\title{
Extraction of primary and secondary frequency control from active power generation data of power plants
}

\author{
B. Ozer ${ }^{a, *}$, O. Arikan ${ }^{a}$, G. Moral ${ }^{a}$, A. Altintas ${ }^{a, b}$ \\ ${ }^{a}$ Department of Electrical and Electronics Engineering, Bilkent University, Ankara, Turkey \\ ${ }^{\mathrm{b}}$ ISYAM, Bilkent University, Ankara, Turkey
}

\section{A R T I C L E I N F O}

\section{Article history:}

Received 20 March 2014

Received in revised form 17 February 2015

Accepted 17 March 2015

Available online 18 April 2015

\section{Keywords:}

Power system monitoring

Power system frequency control

Sparse signal recovery

Signal processing applied to power systems

\begin{abstract}
A B S T R A C T
Frequency control is a vital component of a secure and robust power grid and it ought to be closely monitored. Frequency control consists of two main components; primary and secondary control and their contributions are usually aggregated in the active power generation data of a plant, which is acquired via Supervisory Control And Data Acquisition. In many cases, such as in Turkey, they are demanded to be evaluated separately due to different impacts on power system or different financial policies. However, this is not usually a straightforward process since primary and secondary response cannot be obtained distinctly.

In this work, Extraction of Primary and Secondary Control (EPSCon) algorithm is introduced to extract primary and secondary response over active power generation data. Based on time and frequency domain characteristics of primary and secondary response, EPSCon is developed on a Expectation-Maximization type recursive scheme employing Generalized Cross Correlation and $\ell^{1}$ Trend Filtering techniques. Favorably, EPSCon uses a simple plant model built upon basic governor and plant load controller technical characteristics as an initial estimate of primary and secondary response.
\end{abstract}

(C) 2015 Elsevier Ltd. All rights reserved.

\section{Introduction}

Load-frequency control (LFC), is an essential requirement for a secure and robust power system [1]. Due to increasing size and complexity of power systems [2] and the liberalization of the electricity supply industry [3], it became inevitable to monitor and evaluate frequency control performed by plants. As focused in this paper, primary and secondary frequency control are two main parts of LFC. They have often different remuneration policies [4]. Nevertheless, in a typical Supervisory Control And Data Acquisition (SCADA) application, as in the Turkish system, primary and secondary responses are not acquired distinctly but as an aggregation in the active power generation data of a plant. Using signal processing techniques, this paper introduces Extraction of Primary and Secondary Control (EPSCon) algorithm to extract primary and secondary control components from active power generation data, allowing distinct evaluation and remuneration of primary and secondary control.

LFC aims to stabilize system frequency within limits around nominal frequency by properly adjusting the MW outputs of the

\footnotetext{
* Corresponding author. Tel.: +90 5336839342.

E-mail addresses: ozer@ee.bilkent.edu.tr (B. Ozer), oarikan@ee.bilkent.edu.tr (O. Arikan),gm@ee.bilkent.edu.tr (G. Moral), altintas@ee.bilkent.edu.tr (A. Altintas).
}

generators [5]. Primary frequency control is the automatic response of turbine governors against deviations in system frequency. It depends on the speed-droop characteristics [5] of a plant and performed within a few seconds [6]. Secondary frequency control is dictated by the Automatic Generation Control (AGC) based on the Area Control Error (ACE). For the purposes of this paper, Turkish system is based on a single control area and the system frequency is monitored by the national control center. EPSCon utilizes the reference model introduced in [7] to characterize primary and secondary response of typical power plant in Turkish power system. Secondary frequency control is based on up/down ramp rates of generating plants and realized within the time frame of minutes [8]. In this work, the signals denoting primary and secondary frequency control are referred to as primary and secondary frequency response, respectively.

In the literature, there are many studies regarding optimal load-frequency control strategies, e.g. classical approaches [9-11] or recent techniques [12-16]. Monitoring of power systems draws also attention of many researchers such as estimation of required power generation to balance the load [17], estimation of stability index [18] or assessment of the security of the power system [19]. However, to the best of our knowledge, there is only a limited work [20] on the separate estimation of primary and secondary response components over measured power generation 
data. The motivation behind separate estimation lies on the fact that even though primary and secondary response cannot be individually measured, transmission system operators (TSOs) may have different financial policies. For instance, correct provision of primary and secondary control performed by plants are differently remunerated, or primary and secondary control shall be subject to different penalties, since unavailability of these services may pose different system-wide events. Furthermore, some plants are not obliged to secondary control yet they ought to perform primary control. Primary response component of such plants have to be extracted from total power generation to be able to evaluate primary response for remuneration or penalty.

EPSCon provides separate offline estimates of primary and secondary response of a plant from (total) active power generation data available in SCADA. Time and frequency domain characteristics of primary and secondary response are investigated. Based on the observations that secondary response can be modeled as a piecewise linear signal and it has a much sparser derivative compared to primary response, $\ell^{1}$ trend filter [21] is used to filter out primary response from active power generation. It is possible to improve the estimation performance by initially predicting primary response using the reference model introduced in [7] and subtracted from the active power generation before $\ell^{1}$ trend filter. It is also observed that the reference model provides close estimates of primary response with a time delay and attenuation. Such time delay and attenuation is computed by the correlation between predicted and modeled primary response. A recursive mechanism is used to improve the accuracy of time delay and attenuation computations iteratively. Owing to recursion, better estimates of primary and secondary response are acquired in each iteration. Simulations are provided with synthetic and real data to demonstrate that EPSCon converges to reliable estimates of primary and secondary response after a few iterations.

This paper is organized as follows: In Section 'Nomenclature', signals used in the present research are summarized. In Section 'Time and frequency domain analysis of frequency control', primary and secondary frequency response are analyzed in time and frequency domain, providing constraints for the design procedure of EPSCon. In Section 'Modeled primary and secondary response', a frequency control model is introduced to enhance estimation accuracy. In Section 'EPSCon algorithm', EPSCon algorithm is presented. In Section 'Experimental results', experimental results with both synthetic and real data are illustrated. Finally, conclusions are drawn in Section 'Conclusions'.

\section{Nomenclature}

Table 1 covers the signals that are used either as input or as output in EPSCon.

\section{Time and frequency domain analysis of frequency control}

Analysis of input signals is a crucial step before delving into EPSCon algorithm covered in Section 'EPSCon algorithm'. In this section, both time and frequency domain based analyses are carried out, which are widely used in the design procedure of EPSCon. Active power generation of a plant $\left(P_{G E N}\right)$, sampled by SCADA, is taken to comprise of (actual) primary $\left(\mathrm{PPR}_{A}\right)$ and (actual) secondary frequency response $\left(\mathrm{PSR}_{A}\right)$ as follows:

$P_{G E N}[n]=\operatorname{PPR}_{A}[n]+\operatorname{PSR}_{A}[n]+\omega[n]$,

where $\omega[n]$ is the noise in data which is commonly modeled as a Gaussian variable and $n$ is the discrete time index. Since the signal-to-noise ratio (SNR) is generally very high, $\omega[n]$ will be neglected in the remaining of this work. It should be emphasized
Table 1

Signals and their description used in the presented work.

\begin{tabular}{lll}
\hline Signal & Description & Availability \\
\hline$\Delta f_{S}$ & Deviation in system frequency & SCADA \\
$P_{S E T}$ & Power-set point levels send by AGC & SCADA \\
$P_{G E N}$ & Active power generation of a plant & SCADA \\
$\mathrm{PPR}_{A}$ & (Actual) Primary response of a plant & Unavailable \\
$\widehat{P P R}_{A}$ & Estimated primary response of a plant & Estimated \\
$\mathrm{PPR}_{M}$ & Modeled primary response & Available \\
$\mathrm{PSR}_{A}$ & (Actual) Secondary response of a plant & Unavailable \\
$\widehat{P S R}_{A}$ & Estimated secondary response of a plant & Estimated \\
$\operatorname{PSR}_{M}$ & Modeled secondary response & Available \\
\hline
\end{tabular}

that $\mathrm{PPR}_{A}$ and $\mathrm{PSR}_{A}$ are not individually acquired but their combination $P_{G E N}$ is available through SCADA as a sampled analogue data. In this work, based on $P_{G E N}$ signal, $\mathrm{PPR}_{A}$ and $\mathrm{PSR}_{A}$ are individually estimated, which are represented as $\widehat{\mathrm{PPR}}_{A}$ and $\widehat{\mathrm{PSR}}_{A}$ respectively.

$\mathrm{PPR}_{A}$ is the dynamic response against system frequency $\left(f_{s}\right)$ deviations. Deviations in system frequency $\left(\Delta f_{s}\right)$ is defined as $\Delta f_{s}[n]=f_{u}[n]-f_{s}[n]$, where $f_{u}$ is the nominal frequency which is $50 \mathrm{~Hz}$ for Union for the Co-ordination of Transmission of Electricity (UCTE). Since the generation-load balance of a power system is highly variable in time, $\Delta f_{S}$ and $\mathrm{PPR}_{A}$ are expected to vary rapidly and consequently have considerable amount of high frequency content. In Fig. 1 , an acquired $\Delta f_{s}$ and a representative $\mathrm{PPR}_{A}$ with their spectrum are shown. Spectrum of $\mathrm{PPR}_{A}$ reveals that it has substantially uniform spectrum. Thus, $\mathrm{PPR}_{A}$ cannot be associated with a specific frequency range.

$\mathrm{PSR}_{A}$ is dispatched by power set-point $\left(P_{S E T}\right)$ values which are sent by AGC. $P_{S E T}$ can be regarded as desired active power generation level with the assumption of steady-state $f_{s}$, i.e., $f_{s}[n]=f_{u}[n]$. In this case, excluding primary response, active power generation of a plant should follow $P_{S E T}$, i.e., if $P_{S E T}$ remains constant, active power generation ought to be equal to $P_{S E T}$. Otherwise, power generation of a plant is increased or decreased until $P_{S E T}$ level is attained. Such a behavior is denoted as $\mathrm{PSR}_{A}$. Typically, $\mathrm{PSR}_{A}$ is the response when a plant is controlled by AGC. However, in this work, (1) is assumed to be also valid for plants which are not connected to AGC. In such a case, steady-state active power generation is dictated locally with $P_{S E T}$ levels denoting daily declaration of hourly active power generation schedule. With this extended definition of $\mathrm{PSR}_{A}$ and $P_{S E T}$, it is possible to estimate steady-state active power generation of plants which are only responsible with primary frequency control. As illustrated in Fig. 2, $P_{S E T}$ and $\mathrm{PSR}_{A}$ can be modeled as piecewise linear signals.

Comparison of the spectra of $\mathrm{PPR}_{A}$ and $\mathrm{PSR}_{A}$ shows that frequency content of $\mathrm{PSR}_{A}$ decays faster than $\mathrm{PPR}_{A}$. Average of $\mathrm{PSR}_{A}$ is also much higher than average of $\mathrm{PPR}_{A}$ since $\mathrm{PSR}_{A}$ represents levels of steady-state power generation, it usually has an average
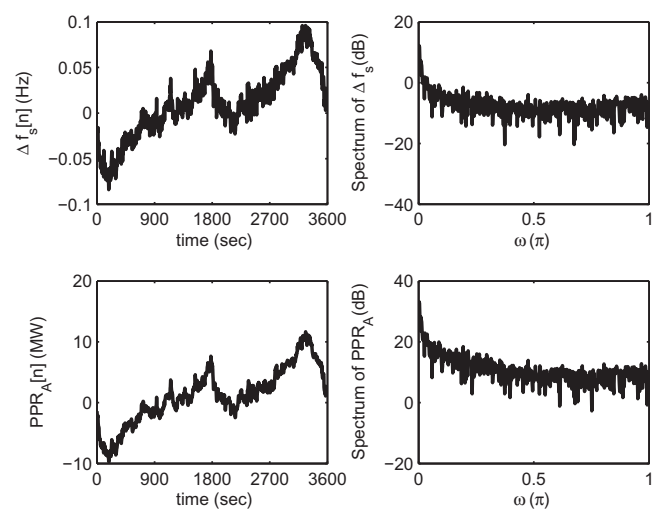

Fig. 1. $\Delta f_{s}, \mathrm{PPR}_{A}$ and their corresponding spectrum 

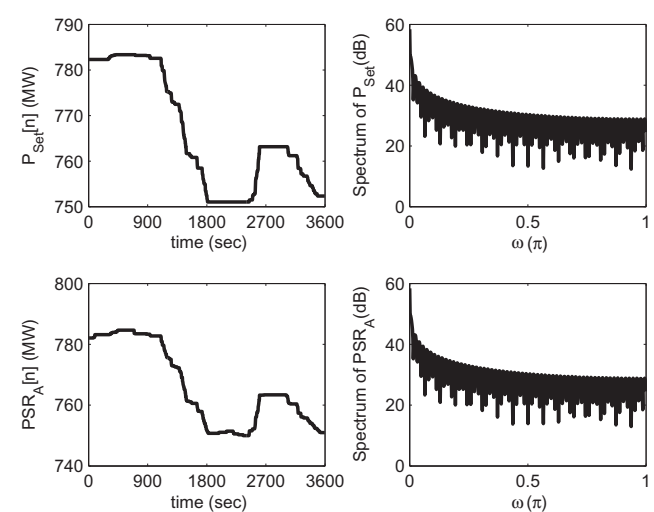

Fig. 2. $P_{S E T}, P_{S R_{A}}$ and their corresponding spectrum.
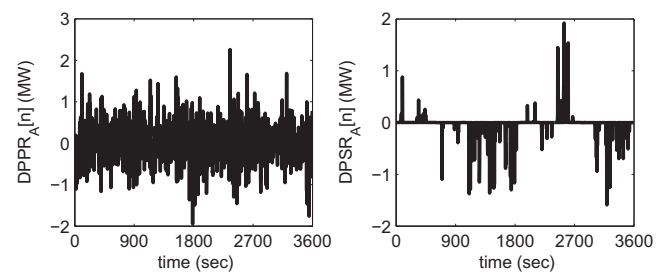

Fig. 3. Derivative of $\mathrm{PPR}_{A}$ and $\mathrm{PSR}_{A}$.

that is close to operational power generation of a plant. Furthermore, as shown in Figs. 1 and 2, $\mathrm{PPR}_{A}$ varies much less frequently than $\mathrm{PSR}_{A}$. Hence, derivative of $\mathrm{PSR}_{A}$ is expected to be much sparser than derivative of $\mathrm{PPR}_{A}$, as illustrated in Fig. 3.

\section{Modeled primary and secondary response}

In the literature, to estimate primary and secondary response, some models are used to represent basic governor and plant load controller technical characteristics. For instance, a reference model presented in [7] provides behaviors of primary and secondary frequency responses that are assumed to fulfill correct provision of frequency control. As illustrated in Fig. 4, modeled $\mathrm{PPR}_{M}$ and $\mathrm{PSR}_{M}$ are computed via blocks which are fed by $\Delta f_{S}$ and $P_{S E T}$, respectively. Internal characteristics (e.g, speed-droop, deadband, up/down ramp rates, etc.) of a plant are also taken into account as detailed in [7].

$\mathrm{PPR}_{M}$ and $\mathrm{PSR}_{M}$ may have considerably different behavior than $\mathrm{PPR}_{A}$ and $\mathrm{PSR}_{A}$, respectively. Still, $\mathrm{PPR}_{M}$ and $\mathrm{PSR}_{M}$ can be regarded as primitive estimates for $\mathrm{PPR}_{A}$ and $\mathrm{PSR}_{A}$. $\mathrm{PPR}_{M}$ may provide a priori information on $\mathrm{PPR}_{A}$, which can be exploited to enhance the accuracy of estimation. Such an idea stems from the fact that primary frequency response is an automatic response against deviations in $f_{S}$ so $\mathrm{PPR}_{M}$ is not expected to be completely independent from $\mathrm{PPR}_{A}$. Many comparisons of measured $\mathrm{PPR}_{A}$ and $\mathrm{PPR}_{M}$ have revealed that they tend to be similar waveforms with different amplitudes. Observations also revealed a presence of possible time shift due to synchronization problems and measurement delays in SCADA. Therefore, in this work, $\mathrm{PPR}_{A}$ is regarded as:

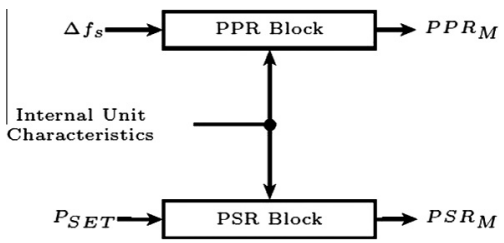

Fig. 4. A reference model for representing primary and secondary response.
$\mathrm{PPR}_{A}[n]=\lambda^{*} \mathrm{PPR}_{M}\left[n-\tau^{*}\right]+\delta \mathrm{PPR}_{A}[n]$,

where $\delta \mathrm{PPR}_{A}$ is an unknown signal representing the difference, $\lambda^{*}$ is the attenuation factor and $\tau^{*}$ is the time delay satisfying:

$\sum_{n}\left(\operatorname{PPR}_{A}[n]-\lambda \operatorname{PPR}_{M}[n-\tau]\right)^{2} \geqslant \sum_{n}\left(\operatorname{PPR}_{A}[n]-\lambda^{*} \operatorname{PPR}_{M}\left[n-\tau^{*}\right]\right)^{2}$.

$\left(\lambda^{*}, \tau^{*}\right)$ pair is considered to be the supreme pair, yielding less or equal difference between $\mathrm{PPR}_{A}$ and $\mathrm{PPR}_{M}$, compared to any other $(\lambda, \tau)$ pair.

Observations on $\mathrm{PSR}_{A}$ and $\mathrm{PSR}_{M}$ indicated that $\mathrm{PSR}_{M}$ has generally a straightforward relation with $\mathrm{PSR}_{A}$ in the form of:

$\operatorname{PSR}_{A}[n]=\operatorname{PSR}_{M}[n]+\delta \operatorname{PSR}_{A}[n]$,

where $\delta \mathrm{PSR}_{A}$ may be regarded as unknown part of $\mathrm{PSR}_{A}$, which is not covered by AGC or daily declaration of hourly active power generation such as violations or internal power consumption of a plant. Furthermore, as in the case of run of river power stations in the French power grid [20], some power plants may not be obliged to declare their daily power generation scheduling nor they are not connected to AGC. In this circumstance, since $P_{S E T}$ is unavailable, $\mathrm{PSR}_{M}$ cannot be computed. In such cases, $\mathrm{PSR}_{M}$ is assumed to be mean of $P_{G E N}$.

\section{EPSCon algorithm}

In Section 'Time and frequency domain analysis of frequency control', $\mathrm{PPR}_{A}$ has been characterized as a signal which has high frequency variations yielding a substantially uniform spectrum whereas $\mathrm{PSR}_{A}$ is a piecewise linear low-pass signal. This situation encourages us to first estimate $\mathrm{PSR}_{A}$ and then to acquire $\mathrm{PPR}_{A}$ by using (1). Let us define $\widehat{\operatorname{PSR}}_{A}$ as:

$\mathrm{PSR}_{A}[n] \triangleq \operatorname{PSR}_{M}[n]+\delta \widehat{\operatorname{PSR}}_{A}[n]$,

where $\delta \widehat{\mathrm{PSR}}_{A}$ is the estimate of $\delta \mathrm{PSR}_{A}$. Then, $\widehat{\mathrm{PPR}}_{A}$ is obtained by: $\widehat{P P R}_{A}[n] \triangleq P_{G E N}[n]-\widehat{\operatorname{PSR}}_{A}[n]$.

Note that estimation of $\delta \mathrm{PSR}_{A}$ yields also an estimate of $\mathrm{PSR}_{A}$ and $\mathrm{PPR}_{A}$ in a progressive order. In this work, $\delta \widehat{P S R}_{A}$ is obtained as: $\delta \widehat{\mathrm{PSR}}_{A}[n] \triangleq L\left\{P_{G E N}[n]-\operatorname{PSR}_{M}[n]-\lambda \operatorname{PPR}_{M}[n-\tau]\right\}$,

\section{Algorithm 1. EPSCon}

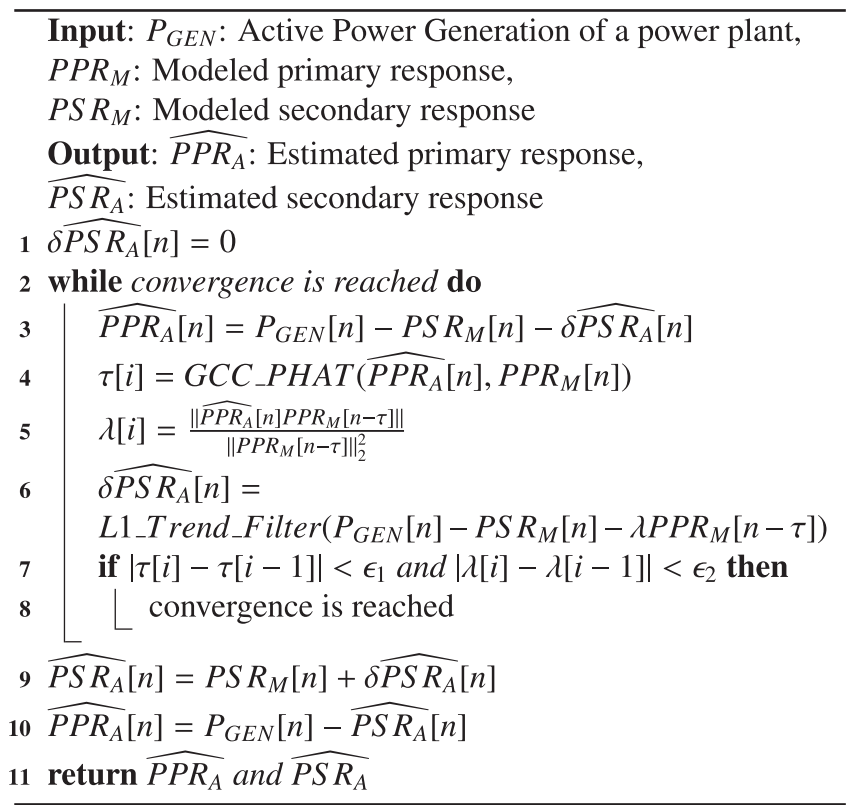


where $L$ is the operator that represents $\ell^{1}$ trend filter introduced in [21], and $(\lambda, \tau)$ is pair computed through Generalized Cross Correlation with Phase Transform (GCC-PHAT) time delay estimation method presented in [22]. EPSCon algorithm is presented in Algorithm 1 and the corresponding block diagram is given in Fig. 5.

The seventh line in Algorithm 1 defines the stopping criteria for preset values of $\epsilon_{1}$ and $\epsilon_{2}$.

The performance of EPSCon is evaluated by following benchmark functions:

$e_{P P R} \triangleq \sum_{n}\left(\operatorname{PPR}_{A}[n]-\widehat{\operatorname{PPR}}_{A}[n]\right)^{2}$,
$e_{P S R} \triangleq \sum_{n}\left(\operatorname{PSR}_{A}[n]-\widehat{\operatorname{PSR}}_{A}[n]\right)^{2}$.

Note that EPSCon yields the same estimation error for both primary and secondary responses:

$$
\begin{aligned}
e_{P P R} & \triangleq \sum_{n}\left(\operatorname{PPR}_{A}[n]-\widehat{\operatorname{PPR}}_{A}[n]\right)^{2} \\
& =\sum_{n}\left(\operatorname{PPR}_{A}[n]-\left(P_{G E N}[n]-\widehat{\operatorname{PSR}}_{A}[n]\right)\right)^{2} \\
& =\sum_{n}\left(\widehat{\operatorname{PSR}}_{A}[n]-\left(\operatorname{P}_{G E N}[n]-\operatorname{PPR}_{A}[n]\right)\right)^{2} \\
& =\sum_{n}\left(\widehat{\operatorname{PSR}}_{A}[n]-\operatorname{PSR}_{A}[n]\right)^{2} \\
& =e_{P S R} .
\end{aligned}
$$

It can be shown that unknown part of (3) can be estimated accurately by (6) in EPSCon. To justify, let us expand (7) by (5):

$$
\begin{aligned}
e_{P P R}= & \sum_{n}\left(\operatorname{PPR}_{A}[n]-\left(P_{G E N}[n]-\widehat{P S R}_{A}[n]\right)\right)^{2} \\
= & \sum_{n}\left(\operatorname{PPR}_{A}[n]-\left(P_{G E N}[n]-\left(\operatorname{PSR}_{M}[n]+\delta \widehat{P S R}_{A}[n]\right)\right)\right)^{2} \\
= & \sum_{n}\left(\operatorname{PPR}_{A}[n]-\left(\operatorname{PPR}_{A}[n]+\operatorname{PSR}_{A}[n]\right)\right)^{2} \\
& \left.\quad+\left(\operatorname{PSR}_{M}[n]+\delta \widehat{P S R}_{A}[n]\right)\right)^{2} \\
= & \sum_{n}\left(\left(\operatorname{PSR}_{M}[n]-\operatorname{PSR}_{A}[n]\right)+\delta \widehat{P S R}_{A}[n]\right)^{2} \\
= & \sum_{n}\left(\operatorname{PSR}_{M}[n]-\left(\operatorname{PSR}_{M}[n]+\delta \operatorname{PSR}_{A}[n]\right)\right. \\
& \left.+\delta \widehat{P S R}_{A}[n]\right)^{2} \\
= & \sum_{n}\left(\delta \widehat{P S R}_{A}[n]-\delta \operatorname{PSR}_{A}[n]\right)^{2},
\end{aligned}
$$

where (4) is used to derive (9a) and (1) and (3) are used to derive (9b) and (9c), respectively. (9d) indicates that error made in the estimation of primary response is associated with the error made in the unknown part of secondary response. (9d) can be further expanded by (6):

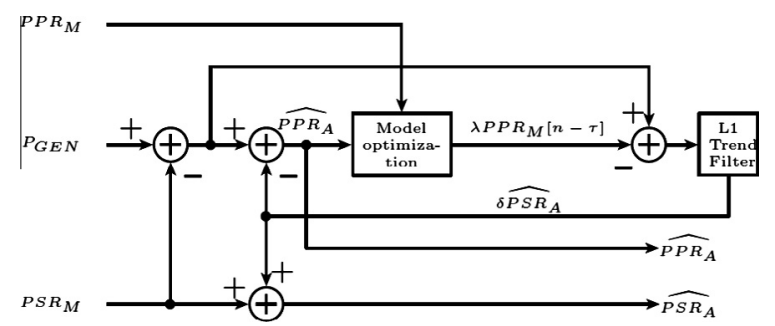

Fig. 5. Block diagram of the estimation scheme for $\mathrm{PPR}_{A}$ and $\mathrm{PSR}_{A}$.

$$
\begin{aligned}
e_{P P R}= & \sum_{n}\left(L\left\{P_{G E N}[n]-\operatorname{PSR}_{M}[n]-\lambda \operatorname{PPR}_{M}[n-\tau]\right\}\right. \\
& \left.-\delta \operatorname{PSR}_{A}[n]\right)^{2} \\
= & \sum_{n}\left(L \left\{\left(\operatorname{PPR}_{A}[n]+\operatorname{PSR}_{A}[n]\right)-\operatorname{PSR}_{M}[n]\right.\right. \\
& \left.\left.-\lambda \operatorname{PPR}_{M}[n-\tau]\right\}-\delta \operatorname{PSR}_{A}[n]\right)^{2} \\
= & \sum_{n}\left(L \left\{\left(\operatorname{PSR}_{A}[n]-\operatorname{PSR}_{M}[n]\right)+\operatorname{PPR}_{A}[n]\right.\right. \\
& \left.\left.-\lambda \operatorname{PPR}_{M}[n-\tau]\right\}-\delta \operatorname{PSR}_{A}[n]\right)^{2} \\
= & \sum_{n}\left(L\left\{\delta \operatorname{PSR}_{A}[n]+r_{P P R}[n]\right\}-\delta \operatorname{PSR}_{A}[n]\right)^{2},
\end{aligned}
$$

where (1) and (3) are used in the (10a) and (10b), respectively. In (10c), $r_{P P R}[n]=\operatorname{PPR}_{A}[n]-\lambda \operatorname{PPR}_{M}[n-\tau]$ is referred to as residual primary response. Note that $e_{P P R}$ is minimized if,

$L\left\{\delta \mathrm{PSR}_{A}[n]+r_{P P R}[n]\right\}=\delta \operatorname{PSR}_{A}[n]$.

The equality is satisfied when the following two conditions are met:

1. $r_{P P R}$ should be eliminated by $L$.

2. $\delta \mathrm{PSR}_{A}$ should pass though $L$ with negligible distortion.

Proper choice of the filter

Conditions 1 and 2 bring significant constraints on the proper choice of the filter $L$. Since $r_{P P R}$ has considerable amount of content in wide spectrum, it is not advisable to design $L$ in frequency domain, e.g., with a specific cut-off frequency. However, it is expected that derivative of (piecewise linear) $\delta \mathrm{PSR}_{A}$ will be much sparser than derivative of $r_{P P R}$ since $\delta \mathrm{PSR}_{A}$ tend to have only limited number of jumps, compared to $r_{P P R}$. Hence, one can solve following minimization problem, using derivative of $\delta \mathrm{PSR}_{A}$ as a Lagrange multiplier:

$\underset{\delta \mathrm{PSR}_{A}}{\min }\left\|\delta \mathrm{PSR}_{A}+r_{P P R}-\delta \widehat{\mathrm{PSR}}_{A}\right\|_{2}^{2}+\alpha\left\|D \delta \widehat{\mathrm{PSR}}_{A}\right\|_{1}^{1}$,

where $D \delta \widehat{P S R}_{A}$ denotes the derivative of $\delta \widehat{\operatorname{PSR}}_{A}$ and $\alpha$ is a regularization parameter used to determine the trade-off between smoothness and the size of residual. Note that using $\ell^{1}$ norm of derivative as a constraint ensures that $\delta \widehat{\mathrm{PSR}}_{A}$ have sparse derivative $[23,21]$. To solve (11), also knowing the shape of $\delta \mathrm{PSR}_{A}$ is in the form of a piecewise linear function, we used $\ell^{1}$ trend filter [21], which yields nonlinear estimate of $\delta \mathrm{PSR}_{A}$ with linear computational time. It has been shown that $\ell^{1}$ trend filter converges in finite number of iterations and error of estimation is bounded by a predefined value, as details can be found in [21].

\section{Expectation maximization type recursion}

Condition 1 is also the fundamental motivation behind the recursion:

$\underset{\lambda, \tau}{\arg \min } \sum_{n} r_{P P R}^{2}[n]=\underset{\lambda, \tau}{\arg \min } \sum_{n}\left(\mathrm{PPR}_{A}[n]-\lambda \mathrm{PPR}_{M}[n-\tau]\right)^{2}=\left(\lambda^{*}, \tau^{*}\right)$,

as $\left(\lambda^{*}, \tau^{*}\right)$ are defined in (2).

$\left(\lambda^{*}, \tau^{*}\right)$ should be derived first for the ideal case $\left(e_{P P R}=0\right) . \tau^{*}$ would be computed by GCC-PHAT algorithm where $\mathrm{PPR}_{A}$ and $\mathrm{PPR}_{M}$ are considered as received form of a signal at two spatially separated sensors. Nonetheless, $\mathrm{PPR}_{A}$ is an also unknown signal that should be estimated. This situation inspires us to use Expectation-Maximization (EM) type recursion as indicated in Fig. 5. Instead of $\mathrm{PPR}_{A}$, at each step, $\widehat{\mathrm{PPR}}_{A}$ is compared with $\mathrm{PPR}_{M}$ 
to estimate $\tau$ according to GCC-PHAT algorithm. Then, $\lambda$ that corresponds to the correlation coefficient is estimated as:

$\lambda=\frac{\sum_{n} \widehat{P P R}_{A}[n] \operatorname{PPR}_{M}[n-\tau]}{\sum_{n} \operatorname{PPR}_{M}^{2}[n-\tau]}$.

This (EM) type scheme provides an iterative way of estimation in which $\delta \widehat{\mathrm{PSR}}_{A}$ and $\delta \widehat{\mathrm{PPR}}_{A}$ are updated at each step, providing more reliable information about each other. This can be deduced by the fact that $\tau$ is obtained by the comparison of $\delta \widehat{P P R}_{A}$ and $\mathrm{PPR}_{M}$. The difference between $\lambda$ and $\lambda^{*}, \tau$ and $\tau^{*}$ are associated with the $e_{P P R}$ since as $e_{P P R}$ is minimized, GCC-PHAT algorithm compares $\mathrm{PPR}_{M}$ with $\delta \widehat{P P R}_{A}$ which is closer to $\mathrm{PPR}_{A}$ than prior case. Therefore, strictly speaking, as long as $(\lambda, \tau)$ pair approach to $\left(\lambda^{*}, \tau^{*}\right), e_{P P R}$ is minimized and vice versa. This situation ensures the convergence of EPSCon. EPSCon is tested both synthetic and real data sets obtained by SCADA and it is observed that $e_{P P R}$ and $e_{P S R}$ tend to decrease at each iteration. EPSCon finally converges to a specific $\delta \widehat{P P R}_{A}$ and $\delta \widehat{P S R}_{A}$ after a couple of iterations. The speed of convergence is generally associated with the amount of $\left\|r_{P P R}\right\|_{2}^{2}$, i.e., how close $\mathrm{PPR}_{A}$ is acquired as a function of $\mathrm{PPR}_{M}$. EPSCon provides an increasing trend of error functions and becomes divergent only when $\mathrm{PPR}_{A}$ has a completely independent behavior than $\mathrm{PPR}_{M}$, which is highly unlikely.

After decomposition of active power generation to its components; primary and secondary responses may be used to evaluate the compliance of the generating plant to the frequency control requirements.

\section{Experimental results}

\section{Synthetic examples}

The performance of EPSCon is tested by synthetically generated $\mathrm{PPR}_{A}$ and $\mathrm{PSR}_{A} . \mathrm{PPR}_{A}$ is selected as $\mathrm{PPR}_{A}[n]=\lambda_{0} \mathrm{PPR}_{M}\left[n-\tau_{0}\right]$ where $\lambda_{0}$ and $\tau_{0}$ are predetermined constants and $P_{G E N}$ is obtained using (1). Then, based on $P_{G E N}, \mathrm{PPR}_{M}$ and $\mathrm{PSR}_{M}, \mathrm{PPR}_{A}$ and $\mathrm{PSR}_{M}$ are estimated. In Fig. 6, inputs for EPSCon algorithm are indicated when no a priori information on $\mathrm{PSR}_{A}$ is available, so $\mathrm{PSR}_{M}$ is selected as the mean of $P_{G E N}$. In Fig. $7, \mathrm{PPR}_{A}$ and $\mathrm{PSR}_{A}$ are compared with $\widehat{P P R}_{A}$ and $\widehat{P S R}_{A}$ at iteration 50, respectively. In Fig. 8, normalized errors for primary and secondary estimates defined as:

$e_{P P R}^{N} \triangleq \frac{e_{P P R}}{\sum_{n} \mathrm{PPR}_{A}^{2}[n]}$,
$e_{P S R}^{N} \triangleq \frac{e_{P S R}}{\sum_{n} \mathrm{PSR}_{A}^{2}[n]}$,

are shown. Both of them are seen to converge quickly.
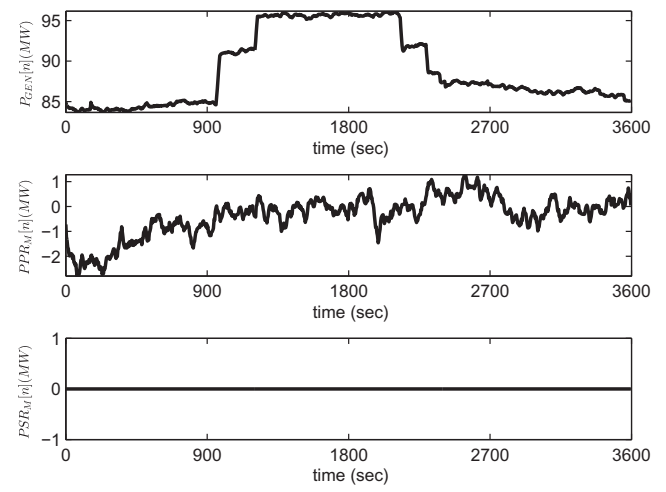

Fig. 6. Synthetic inputs $\left(P_{G E N}, \mathrm{PPR}_{M}\right.$ and $\left.\mathrm{PSR}_{M}\right)$ of EPSCon.

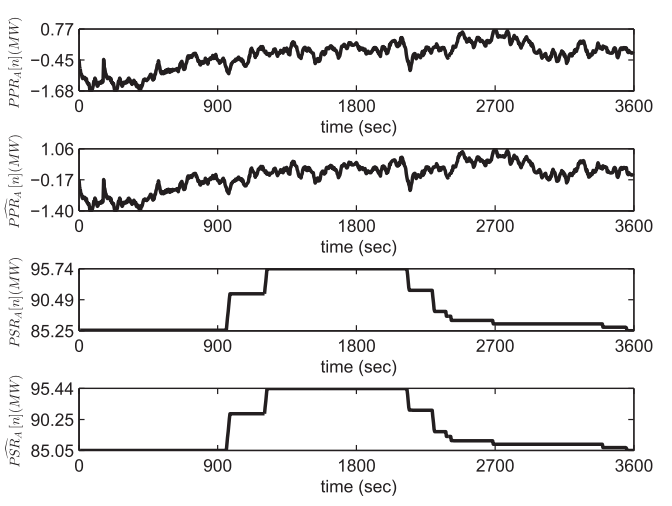

Fig. 7. $\mathrm{PPR}_{A}, \widehat{\mathrm{PPR}}_{A}, \mathrm{PSR}_{A}$ and $\widehat{\mathrm{PSR}}_{A}$ at iteration 50 for the inputs in Fig. 6 .
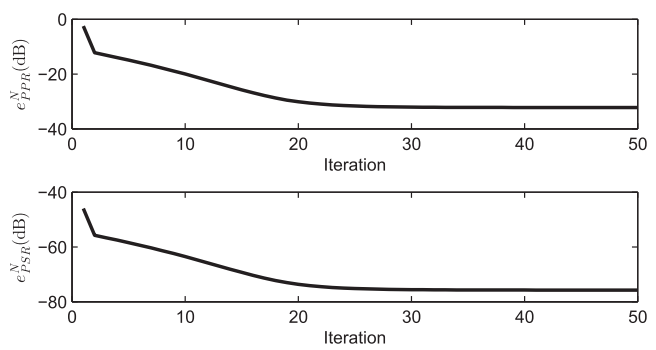

Fig. 8. $e_{P P R}^{N}$ and $e_{P S R}^{N}$ for the inputs in Fig. 6. Both errors converge approximately to zero after a few iterations.

Table 2 compares EPSCon with some other algorithms. $L P$ stands for the algorithm based on low-pass filtering suggested in [20]. 11 th order moving average filter is used as a low-pass filter. SG shows that Savitzky Golay [24] filter is used instead of $\ell_{1}$ trend filter. $F F+\ell_{1}$ indicates the case where no feedback is used, i.e., the results obtained in the first iteration. It has been observed that EPSCon provides much reliable estimates compared to other algorithms.

Results with real data

To illustrate the performance of the EPSCon on real data, $P_{\text {GEN }}$ that is obtained by SCADA/EMS of Turkish Power System is used. Fig. 9 depicts an example in which no information on $\mathrm{PSR}_{A}$ is available, so $\mathrm{PSR}_{M}$ is selected as the mean of $P_{G E N}$. In Fig. $10, \mathrm{PPR}_{A}$ and $\mathrm{PSR}_{A}$ are compared with $\widehat{\mathrm{PPR}}_{A}$ and $\widehat{\mathrm{PSR}}_{A}$ at iteration 50, respectively. Note that $\mathrm{PPR}_{A}$ and $\mathrm{PSR}_{A}$ are unknown signals that are wanted to be estimated. Nevertheless, in Fig. 10, representative $\mathrm{PPR}_{A}$ and $\mathrm{PSR}_{A}$, based on local plant data, are used to discuss the performance of EPSCon. It can be said that $\widehat{P S R}_{A}$ is a reliable estimate of $\mathrm{PSR}_{A}$, although minor errors are observed especially around abrupt variations in $\operatorname{PSR}_{A} . e_{P P R}^{N}$ and $e_{P S R}^{N}$ are shown in Fig. 11.

Minor errors can be eliminated by some a priori information on $\mathrm{PSR}_{A}$. In Fig. 12, $\mathrm{PSR}_{M}$ is assumed to provide an estimate of $\mathrm{PSR}_{A}$ with an offset. When EPSCon is tested with the same configuration

Table 2

Comparison of the algorithms for synthetic data.

\begin{tabular}{lcc}
\hline Method & $e_{P P R}^{N}(\mathrm{~dB})$ & $e_{P S R}^{N}(\mathrm{~dB})$ \\
\hline EPSCon & -32.20 & -75.73 \\
$L P$ & -1.58 & -45.11 \\
$S G$ & -10.05 & -53.57 \\
$F F+\ell_{1}$ & -2.48 & -46.01 \\
\hline
\end{tabular}



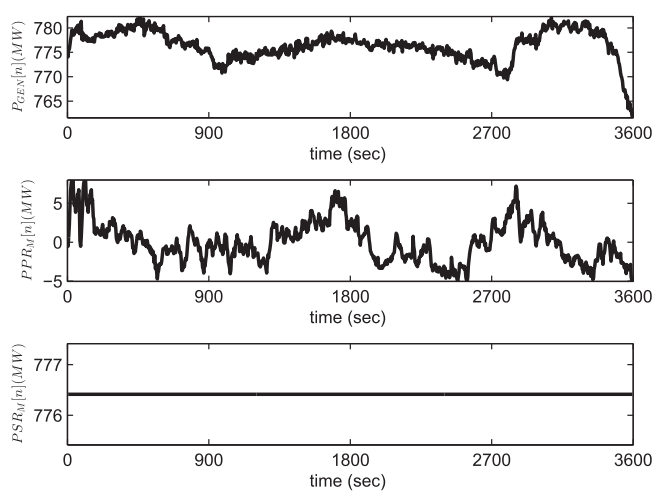

Fig. 9. Real data inputs of EPSCon.

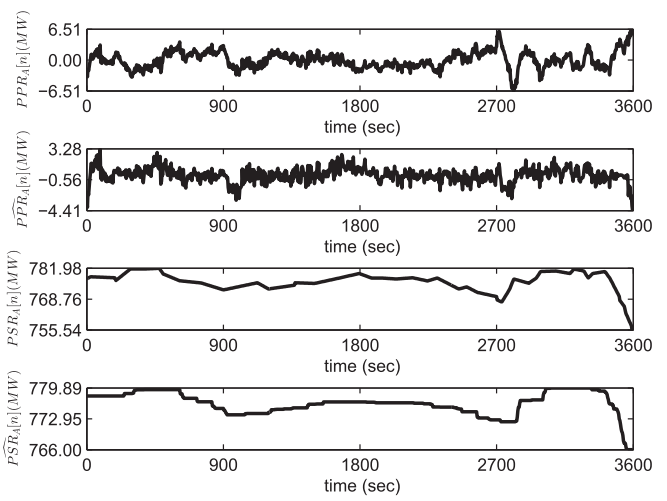

Fig. 10. $\mathrm{PPR}_{A}, \widehat{\mathrm{PPR}}_{A}, \mathrm{PSR}_{A}$ and $\widehat{\mathrm{PPR}}_{A}$ at iteration 50 for the inputs in Fig. 9.
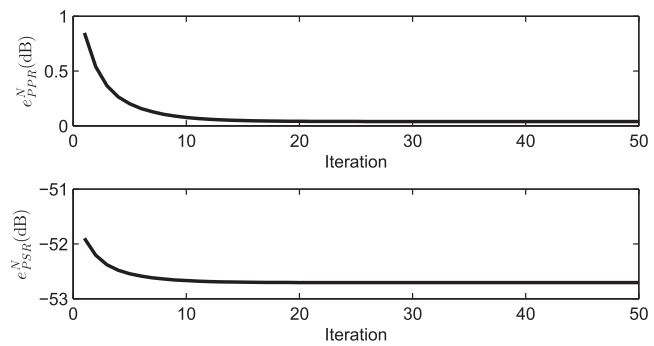

Fig. 11. $e_{P P R}^{N}$ and $e_{P S R}^{N}$ for the inputs in Fig. 9 .

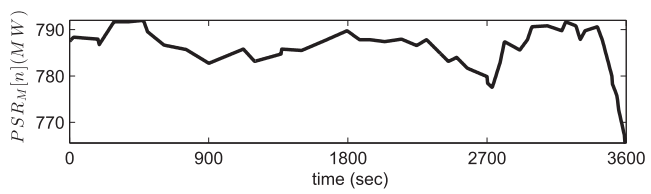

Fig. 12. $\mathrm{PSR}_{M}$ is assumed to provide an estimate of $\mathrm{PSR}_{A}$ with an offset.

given in Fig. 9, with $\mathrm{PSR}_{M}$ as in Fig. 12, $\widehat{\mathrm{PPR}}_{A}$ and $\widehat{\mathrm{PSR}}_{A}$ are acquired as in Fig. 13. It is observed that the accuracy of the estimation is enhanced, minor errors are reduced to negligible level. Fig. 14 indicates $e_{P P R}^{N}$ and $e_{P S R}^{N}$ which are significantly lower than the previously obtained results shown in Fig. 11.

Table 3 tabulates the estimation performance of EPSCon, $L P, S G$ and $F F+\ell_{1}$ for the first case, where the mean of $P_{G E N}$ is used as a priori, as indicated in Fig. 9. It has been observed that although EPSCon makes the least errors, there is no considerable difference

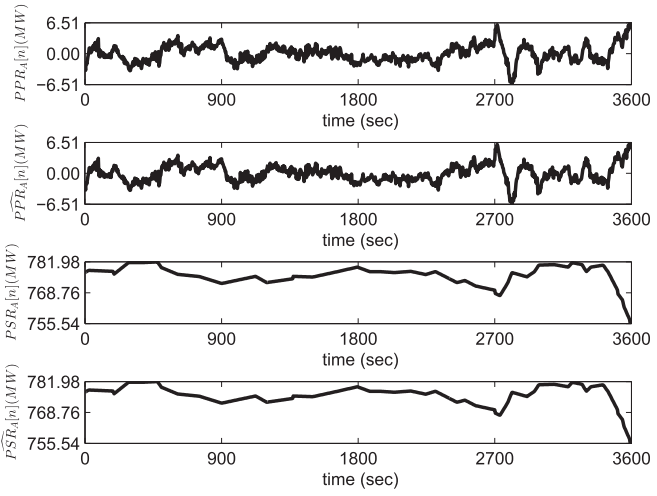

Fig. 13. $\mathrm{PPR}_{A}, \widehat{P P R}_{A}, \mathrm{PSR}_{A}$ and $\widehat{\mathrm{PPR}}_{A}$ at iteration 50 for the inputs in Fig. 9 with known $\mathrm{PSR}_{M}$ as in Fig 12. $\widehat{\mathrm{PPR}}_{A}$ and $\widehat{\mathrm{PPR}}_{A}$ are very reliable estimates of $\mathrm{PPR}_{A}$ and $\mathrm{PSR}_{A}$, respectively.
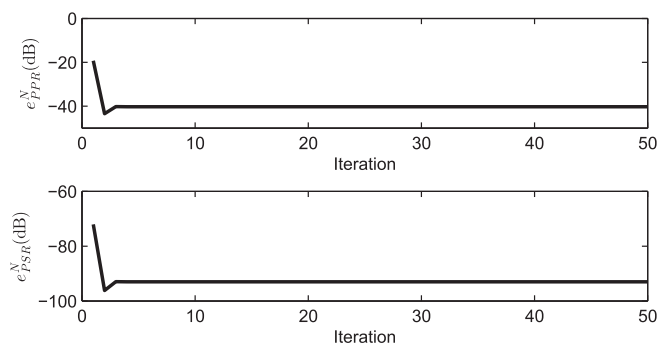

Fig. 14. $e_{P P R}^{N}$ and $e_{P S R}^{N}$ for the inputs in Fig. 9 with known $P_{P R}$ as in Fig. 12.

Table 3

Comparison of the algorithms for read data when no information about $\mathrm{PSR}_{A}$ is available as a priori.

\begin{tabular}{lll}
\hline Method & $e_{P P R}^{N}(\mathrm{~dB})$ & $e_{P S R}^{N}(\mathrm{~dB})$ \\
\hline EPSCon & 0.03 & -52.70 \\
$L P$ & 0.84 & -51.89 \\
$S G$ & 0.37 & -52.36 \\
$F F+\ell_{1}$ & 0.80 & -51.93 \\
\hline
\end{tabular}

Table 4

Comparison of the algorithms for read data when $\mathrm{PSR}_{A}$ is known with an offset.

\begin{tabular}{lrc}
\hline Method & $e_{P P R}^{N}(\mathrm{~dB})$ & $e_{P S R}^{N}(\mathrm{~dB})$ \\
\hline EPSCon & -40.23 & -92.98 \\
$L P$ & -0.85 & -53.60 \\
$S G$ & 0.37 & -52.36 \\
$F F+\ell_{1}$ & -19.29 & -72.03 \\
\hline
\end{tabular}

between algorithms. Table 4, on the other hand, indicates the performance of the algorithms when PSR $_{M}$ provides an estimate of $\mathrm{PSR}_{A}$ with an offset, as illustrated in Fig. 12. As shown in Table 4, all algorithms yield more accurate results when prior information of $\mathrm{PSR}_{A}$ is available. However, $\ell_{1}$ trend filter utilizes such information much better than, a moving average low-pass filter or Savitzky Golay since the derivative of $\delta \widehat{\mathrm{PSR}}_{A}$ becomes much sparser. Hence, EPSCon and $F F+\ell_{1}$ dramatically enhance their performance.

\section{Conclusions}

For reliable extraction of the primary and secondary frequency response components from the active power generation data of a 
plant, a novel algorithm that is called as EPSCon is proposed. $\ell^{1}$ Trend filtering is used to separate primary and secondary frequency whose derivatives have distinct sparsity characteristics. A model of primary response is used to enhance the accuracy of estimation. Model parameters are updated based on previous estimations in a EM type iterative approach.

Detailed performance assessment results of EPSCon in both synthetic and real data sets have indicated that in general EPSCon has provided reliable estimates that have been obtained in a few iteration. However, it has been observed that abrupt variations in the secondary response adversely affect the accuracy of the estimates obtained by EPSCon. To overcome this, a model, that is built upon basic governor and plant load controller characteristics, providing a priori information on primary and secondary response is utilized. Consequently, EPSCon is a highly reliable technique that provides accurate estimates for the primary and secondary responses.

\section{Acknowledgments}

This work was supported by HAVELSAN Company and TEIAS, Turkish Electricity Transmission Company, under project YHMIKS. We also would to thank TEIAS for allowing us to work with real signals acquired by SCADA. We also thank Mr. Oguz Yilmaz at Gama Power Systems Inc. for inspiring discussions.

\section{References}

[1] Kundur P, Balu NJ, Lauby MG. Power system stability and control, vol. 7. New York: McGraw-hill; 1994.

[2] Bevrani H. Robust power system frequency control. Springer; 2009.

[3] Rebours Y, Kirschen D, Trotignon M, Rossignol S. A survey of frequency and voltage control ancillary services; Part I: Technical features. IEEE Trans Power Syst 2007;22(1):350-7.

[4] Rebours Y, Kirschen D, Trotignon M, Rossignol S. A survey of frequency and voltage control ancillary services;part ii: Economic features. IEEE Trans Power Syst 2007;22(1):358-66.

[5] Pandey S, Mohanty S, Kishor N. A literature survey on load-frequency control for conventional and distribution generation power systems. 'Renew Sustain Energy Rev 2013;25:318-34.
[6] Hassan LH, Moghavvemi M, Almurib HA, Steinmayer O. Current state of neural networks applications in power system monitoring and control. Int J Electr Power Energy Syst 2013;51:134-44.

[7] Lobato E, Egido I, Rouco L. Monitoring frequency control in the turkish power system. Electr Power Syst Res 2012;84(1):144-51.

[8] NERC. Balancing and frequency control, NERC, Tech. Rep.; 2011.

[9] Concordia C, Kirchmayer L. Tie-line power and frequency control of electric power systems [includes discussion]. Power Apparatus Syst III. Trans am Inst Electr Eng 1953;72(2).

[10] Feliachi A. Load frequency control using reduced order models and local observers. Int J Energy Syst 1987;7(2):72-5.

[11] Velusami S, Romar K. Design of observer-based decentralized load-frequency controllers for interconnected power systems. Int J Power Energy Syst 1997;17(2):152-60.

[12] Beaufays F, Abdel-Magid Y, Widrow B. Application of neural networks to loadfrequency control in power systems. Neural Netw 1994;7(1):183-94.

[13] Sudha K, Vijaya Santhi R. Robust decentralized load frequency control of interconnected power system with generation rate constraint using type-2 fuzzy approach. Int J Electr Power Energy Syst 2011;33(3):699-707.

[14] Ali R, Mohamed TH, Qudaih YS, Mitani Y. A new load frequency control approach in an isolated small power systems using coefficient diagram method. Int J Electr Power Energy Syst 2014;56:110-6.

[15] Toulabi M, Shiroei M, Ranjbar A. Robust analysis and design of power system load frequency control using the Kharitonov's theorem. Int J Electr Power Energy Syst 2014;55:51-8.

[16] Yesil E, Savran AI, Guzay C. Load frequency controller design using new big bang-big crunch 2 algorithm. In: 2014 IEEE international symposium on innovations in intelligent systems and applications (INISTA) proceedings. IEEE; 2014. p. 7-13.

[17] Moghavvemi M, Yang S, Kashem M. A practical neural network approach for power generation automation. 1998 international conference on energy management and power delivery, 1998. Proceedings of EMPD'98, vol. 1. IEEE; 1998. p. 305-10.

[18] Kamalasadan S, Thukaram D, Srivastava A. A new intelligent algorithm for online voltage stability assessment and monitoring. Int J Electr Power Energy Syst 2009;31(2):100-10.

[19] Seyed Javan D, Rajabi Mashhadi H, Rouhani M. A fast static security assessment method based on radial basis function neural networks using enhanced clustering. Int J Electr Power Energy Syst 2013;44(1):988-96.

[20] Juston P, Guy F, Henry S, Bertolini P, Monitoring of generating units' contribution to frequency and voltage control. In: PowerTech, 2009 IEEE Bucharest, 28 2009-July 2. p. 1-5.

[21] Kim S-J, Koh K, Boyd S, Gorinevsky D. L1 trend filtering; 2007.

[22] Knapp C, Carter GC. The generalized correlation method for estimation of time delay. IEEE Trans Acoust Speech Signal Process 1976;24(4):320-7.

[23] Donoho DL. Compressed sensing. IEEE Trans Inf Theory 2006;52(4):289-1306.

[24] Schafer RW. What is a Savitzky-Golay filter? [lecture notes]. IEEE Signal Process Mag 2011;28(4):11-117. 